\title{
Supersonic Deflagrations in Cosmological Phase Transitions
}

\author{
H. Kurki-Suonio ${ }^{a *}$ and M. Laine ${ }^{b \dagger}$ \\ ${ }^{a}$ Research Institute for Theoretical Physics and ${ }^{b}$ Department of Physics, \\ P.O. Box 9, FIN-00014 University of Helsinki, Finland
}

(5 January 1995)

\begin{abstract}
The classification of the hydrodynamical growth mechanisms for the spherical bubbles of the low-temperature phase in cosmological phase transitions is completed by showing that the bubbles can grow as supersonic deflagrations. Such deflagrations consist of a Jouguet deflagration, followed by a rarefaction wave. Depending on the amount of supercooling, the maximal velocity of supersonic deflagrations varies between the sound and the light velocities. The solutions faster than supersonic deflagrations are weak detonations.
\end{abstract}

PACS numbers: 98.80.Cq, 47.75.+f, 95.30.Lz

*Email: hkurkisu@pcu.helsinki.fi

${ }^{\dagger}$ Email: mlaine@phcu.helsinki.fi 


\section{INTRODUCTION}

When an exothermic process takes place in an ideal fluid, knowing the hydrodynamics of growing bubbles becomes relevant. Such situations include spherically symmetric chemical burning in combustible gases [1,2], and the growth of bubbles of the low-temperature phase in cosmological first-order phase transitions [3] [13]. The solutions of the energy-momentum conservation equations across the 'burning' surface can naturally be divided into six hydrodynamical modes with different qualitative features, called the weak, strong, and Jouguet deflagrations, and the weak, strong, and Jouguet detonations. When stability considerations, the microscopic mechanism of entropy production at the burning surface, and the overall boundary conditions, are taken into account, it turns out that some of the hydrodynamical modes are actually forbidden. It is the purpose of the present paper to complete the existing classification of the allowed hydrodynamical modes in cosmological phase transitions, and to present, to our knowledge, a new flow profile by which the burning surface can be connected to the boundary conditions.

Our new results concern deflagrations, which are the slower of the two main types of solutions. Usually, it is assumed that the flow profile of a deflagration bubble is such that the burning surface leaves the matter at rest. Then weak deflagrations are subsonic and Jouguet deflagrations expand at sound velocity. The strong deflagrations would be supersonic, but it is known for chemical burning that such solutions do not exist [1,2]. We find that the strong deflagrations are forbidden in cosmological phase transitions, as well. However, there is another type of a supersonic mode. Indeed, a Jouguet deflagration can also leave behind it a rarefaction wave, in which case matter is not at rest immediately behind the burning surface. Such solutions are supersonic, and fill in the velocity gap between the sound velocity and the slowest detonations. As far as we can see, supersonic deflagrations should be possible both in chemical burning, and in cosmological phase transitions, such as the QCD and the electroweak phase transition.

The plan of the paper is the following. In Sec. II, the properties of bubble growth 
in relativistic ideal fluid are reviewed both for deflagrations and detonations. In Sec. III, we use a simple dynamical model to study which hydrodynamical growth mechanisms can actually be reached from an initial critical bubble configuration. In particular, supersonic deflagrations are found to be possible. The structure of spherical supersonic deflagration bubbles is analyzed in detail in Sec. $\mathbb{\nabla}$, for the bag equation of state. The conclusions are in Sec. $\mathrm{V}$.

\section{DEFLAGRATIONS AND DETONATIONS}

Let us go into the rest frame of the expanding phase transition surface. Imposing energymomentum conservation across the discontinuity gives the equations

$$
\begin{gathered}
w_{q} \gamma_{q}^{2} v_{q}=w_{h} \gamma_{h}^{2} v_{h} \\
w_{q} \gamma_{q}^{2} v_{q}^{2}+p_{q}=w_{h} \gamma_{h}^{2} v_{h}^{2}+p_{h}
\end{gathered}
$$

where the subscripts $q$ and $h$ refer to the inflowing high-temperature ('quark') phase and the out-flowing low-temperature ('hadron') phase, respectively ( $w$ is the proper enthalpy density). We choose the signs so that the velocities are positive, and denote by $F$ the quantity $w_{q} \gamma_{q}^{2} v_{q}$. It then follows that

$$
F=\frac{p_{h}-p_{q}}{v_{q}-v_{h}}>0
$$

This equation tells us that there are two qualitatively different kinds of solutions. For deflagrations, the pressure decreases and the velocity increases across the surface: $p_{h}<p_{q}$ and $v_{h}>v_{q}$. For detonations, the opposite is true. For deflagrations, the inflow velocity $v_{q}$ is subsonic, and for detonations, it is supersonic [1,14. Note that for deflagrations, the sign

of the pressure difference is opposite to what it would have been, had the temperature not changed across the phase transition surface.

A further division of the different hydrodynamical processes can be made according to the outflow velocity $v_{h}$. Both for deflagrations and detonations, energy-momentum conservation 
allows $v_{h}$ to be on either side of, or equal to, the sound velocity $c_{s}$. If $v_{h}$ equals $c_{s}$, the process is called a Jouguet process. If $v_{h}$ is on the same side of $c_{s}$ as $v_{q}$, the process is called weak; otherwise it is called strong. For instance, for weak deflagrations, $v_{h}<c_{s}$.

To construct a complete bubble solution, the discontinuity at the phase transition surface has to be matched with possible shock discontinuities and continuous flow profiles, to satisfy the boundary conditions. A deflagration solution can be constructed from a supersonic shock front preceding the phase transition surface and setting the cosmic matter moving, followed by a continuous flow profile, and terminated by the phase transition front bringing the matter back at rest. A detonation solution can be constructed from a weak or Jouguet detonation front moving supersonically into matter at rest, and followed by a continuous rarefaction wave bringing the matter again at rest. With a strong detonation, the boundary conditions of a spherically expanding bubble cannot be satisfied [1,3]. Flow profiles of deflagration bubbles are given in Ref. [5], and flow profiles of detonation bubbles are illustrated, e.g., in Fig. 2 of Ref. [14]. These simplest complete flow profiles are dubbed, according to the nature of the phase transition front, the weak, Jouguet and strong deflagrations, and the weak and Jouguet detonations. In the next Section, it is seen that the presented solutions do not quite exhaust the flow profiles relevant for cosmological phase transitions. In the remainder of this Section, on the other hand, it is argued that some of the solutions will not be realized in nature.

First, strong deflagrations are expected to be forbidden. For chemical combustion, there are at least two ways of seeing this. Assuming that the burning front can be described as a changing mixture of the burnt and the unburnt matter, one can see that strong deflagrations would include regions of negative entropy production [2, [14], and they are therefore impossible. This proof is not valid for cosmological phase transitions, since the assumption of a changing mixture would lead to a vanishing surface tension at $T_{c}$. There is another way of excluding the strong deflagrations, coming from the observation that even if such a solution could momentarily be forced to exist, it would be unstable and tend to split into other solutions [1]. This argument should hold also for cosmological phase transitions. Hence we 
expect to see only weak and Jouguet deflagrations. These expand at most at sound velocity.

Second, consider weak detonations. To study them, one must know something about the microscopic mechanism of phase separation. For chemical combustion [1, 2], the rate of burning rises dramatically with temperature, and the velocity of the burning front is determined by how fast heat can be supplied to yet unburnt regions. As a consequence, the microscopic structure of a detonation front is such that a shock front heats the matter so much that rapid burning can begin, and a layer where the burning takes place follows just behind the shock front巴. For weak detonations, the layer behind the shock front is a strong deflagration. Hence, the impossibility of strong deflagrations implies the impossibility of weak detonations. For cosmological phase transitions, the structure of the burning front is different. A phase transition is more apt to start at a low temperature, when supercooling is larger. In particular, a point in space outside the original critical bubble does not wait for a rise in temperature to start transforming into the new phase, but the transformation is caused by interactions with the new phase in the detonation front. Hence, the structure of a detonation front need not be a shock front followed by a deflagration, and weak detonations cannot be excluded in cosmological phase transitions [14].

Let us note that there exist processes in classical hydrodynamics which have the same classification of the different hydrodynamical growth mechanisms as cosmological phase transitions, unlike chemical burning. Such are condensation discontinuities [1, §132]. An example of a condensation discontinuity is the condensation of supersatured water vapour

\footnotetext{
${ }^{1}$ This assumption was also made by Steinhardt in Ref. [3]. In Ref. [3] weak detonations were excluded from consideration by the statement "The lower point $b$ at which the chord ad intersects the detonation adiabat cannot be reached if the reaction is exothermic", which assumes the detonation is initiated by a shock. Ref. [3] is often quoted as a proof that Jouguet detonations are the only kinds of detonations that occur, but this is a result of relativistic combustion theory, which does not apply to cosmological phase transitions.
} 
into small water droplets, i.e., fog. This is a phase transition, though with a finite chemical potential. Indeed, only strong deflagrations and detonations are excluded in Ref. [1], and the other processes are accepted.

\section{NUMERICAL RESULTS}

To be able to check the presented arguments about the possibility of the different kinds of solutions, one would like to have a way of taking automatically into account energymomentum conservation, boundary conditions, and the microscopic structure of the phase transition surface. One might also wish to follow the real-time history of the growing bubbles, to see which stationary final states can actually be reached from reasonable initial conditions. These problems can be solved by imposing an equation of motion for some continuously changing order parameter field $\varphi(t, \mathbf{x})$, and by then solving the equation. The equation could, in principle, be derived from non-equilibrium field theory.

In Ref. [15], the simple phenomenological form

$$
\partial^{\mu} \partial_{\mu} \varphi+\frac{\partial V}{\partial \varphi}+\eta u^{\mu} \partial_{\mu} \varphi=0
$$

of the equation for the $\varphi$-field was proposed (in Ref. [15] the notation $1 / \Gamma$ was used for

$\eta)$. This is the simplest relativistic generalization of the Langevin equation without the noise term. The parameter $\eta$ fixes the one degree of freedom left free by energy-momentum conservation equations. From eq. (4) and energy-momentum conservation it follows that the entropy density $s$ satisfies

$$
T \partial_{\mu}\left(s u^{\mu}\right)=\eta\left(u^{\mu} \partial_{\mu} \varphi\right)^{2}
$$

so that $\eta$ can be interpreted as a parametrization of the entropy production at the phase transition surface. Below, $\eta$ is treated as a free parameter, although for the EW phase transition, its value could be estimated [15] by comparing the analytic formula for the deflagration

front velocity in the limit of small supercooling with microscopic calculations [16] 18$]$ of the same quantity. 
In Ref. [15], eq. (44) and the energy-momentum conservation equations were solved numerically for the planar symmetric case, corresponding to a $1+1$ dimensional spacetime. Note that the dimension of space $d$ does not affect the results qualitatively, since the phase transition front looks locally planar, and the qualitative features of the continuous flow profiles outside the phase transition front do not depend on $d$ [5]. There are two methods of solving eq. (4). Either one can take some initial bubble, and integrate all the partial differential equations forward in time (the dynamical code), or one can search only for stationary final states (the static code).

The general structure of the stationary final states is shown in Fig. 11. In this figure, three kinds of solutions are displayed. First, the dotted lines $(\alpha, \ldots)$ each represent a oneparameter family of solutions, with a given initial supercooling $T_{f}$, which satisfy the energymomentum conservation equations and the boundary conditions, alone. In drawing these curves, it was assumed that the solutions are of the type discussed in Sec. П1, Second, the solid lines represent the solutions which satisfy energy-momentum conservation and eq. (田) across the phase transition surface, with a given $\eta$, but for which the global boundary conditions have not been imposed. For given $T_{f}$ and $\eta$, the possible stationary final states are then found from the intersections of these two kinds of curves. Third, the gray lines indicate those stationary final states which are actually realized by the dynamical code, starting from a reasonable initial state, for given $T_{f}$, as a function of $\eta$. As illustrated with the arrows for the curve $\alpha$, the solution is a weak deflagration in the limit $\eta \rightarrow \infty$, moves towards strong deflagrations as $\eta$ is decreased, and changes to a detonation at some point.

A few interesting things can be seen in Fig. 1. First, one should note the general feature that weak detonations exist, as anticipated in Sec. ㅍ. Second, the dotted lines have a very large gradient for deflagrations. This indicates that the temperature $T_{q}$ in front of the phase transition surface may be much higher than $T_{f}$ due to the heating caused by the shock front, but the temperature $T_{h}$ behind the phase transition surface is very near $T_{f}$. For detonations, similarly, it turns out that the temperature in the center of the expanding bubble differs much less from $T_{f}$ than the temperature $T_{h}$ just behind the detonation front. Third, it 
is seen that for some $T_{f}$ and $\eta$, there are more than one possible stationary final states. For instance, for $\eta=0.010 T_{c}$ and $T_{f}=0.98 T_{c}$, there are three possible final states. The dynamical code selects one of these'2. The solutions which are not realized have a sharp peak in temperature expanding at roughly the speed of sound. If one gives the unreached weak deflagration as an initial condition to the dynamical code, it remains as such, indicating that this solution may be reachable from other initial conditions. However, the unreached weak detonation transforms to either of the other solutions, suggesting an instability.

Let us now turn to the existence of supersonic deflagrations. From Fig. 1, it is seen that strong deflagrations have not been found with the static code. Technically, this is due to the fact that no matter how well one tries to guess the correct solution, the guess does not relax to a strong deflagration but rather changes considerably to form a weak detonation, with the same $T_{h}$. This is in accordance with strong deflagrations being unstable, as argued in the previous Section. On the other hand, with the dynamical code, we have found supersonic solutions which lie very near the dotted lines representing strong deflagrations (see curve $\alpha$ ). A careful inspection reveals, however, that in the region covered by the lighter shade, the gray curve is not exactly on the dotted curve. By drawing a figure of the temperature profiles, the reason becomes apparent. In Fig. 2, temperature profiles are shown for the supercooling $T_{f}=0.80 T_{c}$. The supersonic deflagration solution $\left(\eta=0.17 T_{c}\right)$ is seen to be comprised of a shock front, a phase transition front, and a rarefaction wave in the lowtemperature phase. If the temperature just behind the phase transition front is taken as the $x$-coordinate in Fig. 1 instead of the temperature in the center of the bubble, the solution lies on the dashed curve corresponding to a Jouguet deflagration. Furthermore, if one gives a strong deflagration as an initial condition to the dynamical code, the solution is unstable, and transforms into a Jouguet deflagration followed by a rarefaction wave. In the next

\footnotetext{
${ }^{2}$ Figs. 19 and 20 of Ref. 15] represent one solution of the static code. The solution of the dynamical code actually changes into a weak detonation at around $\eta=0.03 T_{c}$, with $v_{\text {det }} \approx 0.7$.
} 
Section, these solutions are analyzed in more detail in $1+3$ dimensions.

\section{SPHERICAL SOLUTIONS}

For an overview of the different hydrodynamical solutions for spherical phase transition bubbles, we employ a simpler equation of state, the bag EOS

$$
\begin{aligned}
p_{h} & =\frac{1}{3} \epsilon_{h}, \\
p_{q} & =\frac{1}{3}\left(\epsilon_{q}-4 B\right),
\end{aligned}
$$

where $B$ is the bag constant ( $\epsilon$ is the proper energy density). We assume a spherically symmetric similarity solution, i.e., it can be described in terms of the single coordinate $\xi \equiv r / t$. In contrast to the previous Section, we are here not interested in how the bubble relaxes to the similarity solution from some initial condition. We also give up modelling the

microscopic structure of the phase transition front, and treat it as a discontinuity. Therefore we do not have a parameter $\eta$ coming from microphysics to select among the different solutions hydrodynamically allowed. We just parametrize the solutions by the velocity of the phase transition front $\xi_{\text {p.t. }}\left(\xi_{\text {defl }}\right.$ or $\left.\xi_{\text {det }}\right)$. Then we get almost analytical $(i . e .$, involving ordinary differential equations) solutions for the bubble structure, instead of having to resort to numerical hydrodynamics.

The continuous parts of the flow satisfy the equations [5]

$$
\left[v^{2}\left(3-\xi^{2}\right)-4 v \xi+3 \xi^{2}-1\right] \frac{d v}{d \xi}=2 \frac{v}{\xi}\left(1-v^{2}\right)(1-\xi v)
$$

and

$$
\frac{1}{\tilde{\epsilon}} \frac{d \tilde{\epsilon}}{d \xi}=4 \frac{\xi-v}{1-\xi v} \frac{1}{1-v^{2}} \frac{d v}{d \xi}
$$

where $\tilde{\epsilon}=\epsilon$ in the $h$ phase and $\tilde{\epsilon}=\epsilon-B$ in the $q$ phase.

In Fig. 3 we show a sequence of profiles for phase transition bubbles, with the same initial energy density $\epsilon_{f}$, but for different $\xi_{\text {p.t. }}$. 
If the phase transition front is subsonic, $\xi_{\text {p.t. }}=\xi_{\text {deff }} \leq c_{s}$, we have a deflagration bubble of the type described in [5]. The phase transition front is preceded by a shock at $\xi_{\mathrm{sh}}>c_{s}$, the matter is at rest at $\xi>\xi_{\text {sh }}$ and at $\xi<\xi_{\text {deff }}$, and the shocked fluid in between has a continuous flow profile, with the (outward) flow velocity and energy density increasing with decreasing $\xi$. We denote by $\xi_{\text {sh,J }}$ the shock velocity of the Jouguet deflagration bubble with $\xi_{\text {p.t. }}=c_{s}$. The slowest deflagrations have extremely weak shocks at $\xi_{\mathrm{sh}} \sim c_{s}$.

The slowest detonation is the Jouguet detonation, with

$$
\xi_{\mathrm{det}, J}=\frac{1+3 \sqrt{\frac{B}{2 \epsilon_{f}+B}}}{1+\sqrt{\frac{B}{2 \epsilon_{f}+B}}} c_{s} .
$$

Solutions with $\xi_{\text {p.t. }}>\xi_{\text {det,J }}$ are weak detonations.

The velocity range between $\xi_{\text {p.t. }}=c_{s}$ and $\xi_{\text {p.t. }}=\xi_{\text {det }, \mathrm{J}}$ is covered by the supersonic deflagrations. As $\xi_{\text {p.t. }}$ is increased past $c_{s}$, a rarefaction wave appears in the region between $\xi=c_{s}$ and $\xi=\xi_{\text {p.t. }}$. The phase transition remains a Jouguet deflagration, and is preceded by a shock, with $\xi_{\text {p.t. }}<\xi_{\text {sh }}<\xi_{\text {det,J. }}$. As $\xi_{\text {p.t. }}$ is increased, $\xi_{\text {sh }}$ grows also, but not as rapidly, and thus the region of shocked fluid becomes narrower. The two velocities $\xi_{\text {p.t. }}$ and $\xi_{\text {sh }}$ become equal at $\xi_{\text {p.t. }}=\xi_{\text {sh }}=\xi_{\text {det,J }}$, the region of shocked fluid disappears and the solution becomes a Jouguet detonation.

In the limit where $\xi_{\text {p.t. }}$ approaches $\xi_{\text {det,J }}$ from below, the shocked region becomes infinitesimally thin. This resembles the microscopic structure of a detonation front in chemical combustion, where heating by a shock initiates the transition. However, the weak detonations $\left(\xi_{\text {p.t. }}>\xi_{\text {det,J }}\right)$ here do not have such structure.

In Fig. 1 we show the structure of a supersonic deflagration bubble. Figs. 5 and 6 show how the different quantities in the bubble change as a function of $\xi_{\text {p.t. }}$. Fig. 7 shows the limiting velocities $\xi_{\mathrm{sh}, \mathrm{J}}$ and $\xi_{\mathrm{det}, \mathrm{J}}$ as a function of $\epsilon_{f} / B$.

Let us note that in this Section we have studied pure hydrodynamics, whereas one should actually also insist on entropy production being non-negative at the phase transition front [4]. This requires knowledge of the dimensionless parameter $r=a_{q} / a_{h}$ in the bag EOS $\left(\epsilon_{h}=3 a_{h} T^{4}, \epsilon_{q}=3 a_{q} T^{4}+B\right)$. The parameter $r$ fixes the energy density $\epsilon / B=(4 r-1) /(r-1)$ 
corresponding to the critical temperature $T_{c}$. The entropy condition restricts the availability of the different hydrodynamical modes near $T_{c}$, and for a sufficiently small supercooling, only weak deflagrations remain allowed [19, Sec. IV]. However, considering any $\epsilon_{f}$, for a sufficiently small $r$ this value of $\epsilon_{f}$ will correspond to a large supercooling, and all the hydrodynamical modes are allowed. Therefore the entropy condition does not change our conclusions.

\section{CONCLUSIONS}

We have discovered a new kind of a bubble solution for cosmological phase transitions. The phase transition front of such a bubble is a Jouguet deflagration, is preceded by a shock, and followed by a rarefaction wave. The phase transition front moves supersonically with respect to the matter at rest. These supersonic deflagration bubbles fill the velocity gap between weak deflagrations, which are subsonic, and weak detonations. The two limiting cases of the supersonic deflagration bubble are the 'ordinary' Jouguet deflagration bubble (i.e., without a rarefaction wave) and the Jouguet detonation bubble.

It has been known that strong deflagrations would not occur since they are unstable to 1-dimensional perturbations (i.e., in the radial direction). By following a strong deflagration bubble with a numerical hydrodynamics code we have seen how such a perturbation transforms the strong deflagration into a Jouguet deflagration by developing a rarefaction wave. This gives rise to the supersonic deflagration bubble we have described.

By modelling the microscopic mechanism of entropy production at the phase transition front by a simple dynamical model, we found that the solutions 'near' Jouguet detonations [e.g., profiles 7-9 in Fig. 3] were in many cases not realized in a numerical evolution, although they were allowed by the external conditions (including the entropy condition). The velocity $\xi_{\text {p.t. }}$ of the phase transition front was found not to increase continuously as the microscopic entropy-production parameter $\eta$ was decreased, but the solution jumped from a weak deflagration to a much faster weak detonation. Thus the most 'violent' solutions, i.e., the ones with large energy densities and flow velocities occurring near the phase transition 
front, were skipped over. This happened in the cases with a small supercooling, and we had to go to large supercooling to see examples of supersonic deflagrations. Examining Fig. [1, one finds for small supercooling that, for a given value of $T_{f}$ and $\eta$ there can be as many as three solutions allowed, not just one. One of these is a deflagration, and two are detonations. In the numerical evolution, the weaker, i.e., the faster, of the detonations was then realized.

This suggests that in nature the more dramatic solutions, the Jouguet detonation and solutions near it, are more difficult to realize, especially for small supercooling. This is in sharp contrast to chemical burning, where the Jouguet detonations are the only possible detonation solutions.

\section{ACKNOWLEDGEMENTS}

We are grateful to J. Ignatius for discussions, and to the Finnish Center for Scientific Computing for computational resources. 


\section{REFERENCES}

[1] L. D. Landau and E. M. Lifshitz, Fluid Mechanics, 2nd edition (Pergamon Press, Oxford, 1987).

[2] R. Courant and K. O. Friedrichs, Supersonic flow and shock waves (Springer-Verlag, Berlin, 1985).

[3] P. J. Steinhardt, Phys. Rev. D 25 (1982) 2074.

[4] M. Gyulassy, K. Kajantie, H. Kurki-Suonio, and L. McLerran, Nucl. Phys. B 237 (1984) 477.

[5] H. Kurki-Suonio, Nucl. Phys. B 255 (1985) 231.

[6] K. Kajantie and H. Kurki-Suonio, Phys. Rev. D 34 (1986) 1719.

[7] J. C. Miller and O. Pantano, Phys. Rev. D 40 (1989) 1789, D 42 (1990) 3334.

[8] K. Enqvist, J. Ignatius, K. Kajantie, and K. Rummukainen, Phys. Rev. D 45 (1992) 3415.

[9] S. A. Bonometto and O. Pantano, Phys. Rep. 228 (1993) 175.

[10] P. Huet, K. Kajantie, R.G. Leigh, B.-H. Liu, and L. McLerran, Phys. Rev. D 48 (1993) 2477.

[11] M. Kamionkowski, A. Kosowsky, and M. S. Turner, Phys. Rev. D 49 (1994) 2837.

[12] A. F. Heckler, preprint astro-ph/9407064 (1994).

[13] J. C. Miller and L. Rezzolla, SISSA preprint 161/94/A-EP (1994).

[14] M. Laine, Phys. Rev. D 49 (1994) 3847.

[15] J. Ignatius, K. Kajantie, H. Kurki-Suonio, and M. Laine, Phys. Rev. D 49 (1994) 3854.

[16] M. Dine, P. Huet, R.G. Leigh, A.D. Linde, and D. Linde, Phys. Rev. D 46 (1992) 550. 
[17] B.-H. Liu, L. McLerran, and N. Turok, Phys. Rev. D 40 (1992) 2668.

[18] S.Yu. Khlebnikov, Phys. Rev. D 46 (1992) 3223.

[19] J. Ignatius, K. Kajantie, H. Kurki-Suonio, and M. Laine, Phys. Rev. D 50 (1994) 3738. 


\section{FIGURES}

FIG. 1. The solutions of the model of Ref. [15], in the case of planar symmetry. In $1+3$ dimensions, the solid lines remain the same, but the exact functional form of the dotted lines changes a little. For detonations, the $x$-axis is the temperature just behind the phase transition surface; for deflagrations, it is the temperature in the center of the expanding bubble. The $y$-axis is the temperature just in front of the phase transition surface. The region forbidden kinematically, by the non-negativity of entropy production, or by boundary conditions, has been covered with the darker shade, assuming the types of solutions discussed in Sec. II. The two allowed regions represent the deflagrations and the weak detonations. Dashed lines indicate the Jouguet processes, and strong deflagrations are covered with the lighter shade. The meaning of the different curves has been explained in the text. The nucleation temperatures $T_{f} / T_{c}$ corresponding to $\alpha, \beta, \gamma$, $\delta$ and $\epsilon$ are $0.86,0.89,0.92,0.95$ and 0.98 . The (QCD-type) parameters used in this figure are $L=0.1 T_{c}^{4}, \sigma=0.1 T_{c}^{3}$ and $l_{c}=6 T_{c}^{-1}$. Given the expansion rate of the Universe, the actual nucleation temperature $T_{f} / T_{c}$ is then about 0.86 (Fig. 1 in Ref. 19]).

FIG. 2. Temperature profiles at $t=14400 T_{c}^{-1}$ for different values of $\eta$. All the profiles are moving to the right. The arrows indicate the location of the phase transition front. For $\eta=0.20 T_{c}$, the solution is a weak deflagration; for $\eta=0.17 T_{c}$, it is a Jouguet deflagration followed by a rarefaction wave; for $\eta=0.16 T_{c}$ and $\eta=0.12 T_{c}$, the solution is a weak detonation. The velocity of the phase transition front changes from 0.56 at $\eta=0.20 T_{c}$ to 0.74 at $\eta=0.12 T_{c}$.

FIG. 3. Energy-density (upper set) and flow velocity (lower set) profiles for spherical phase transition bubbles. We show 13 profiles, all for the same initial energy density $\epsilon_{f}=100 B$, but with different phase transition front velocities. The six slowest (on the left) are weak deflagrations. The seventh is a Jouguet deflagration bubble, with $\xi_{\text {defl }}=c_{s}$. The eighth is a supersonic deflagration bubble, which has a Jouguet deflagration front preceded by a shock and followed by a rarefaction wave. The ninth is a supersonic deflagration bubble almost at the limit where it becomes a Jouguet detonation. The shocked region is now so thin that it appears as a mere vertical line in the figure. The last four (on the right) are weak detonations. 
FIG. 4. A supersonic deflagration bubble. The upper curve is the energy density profile and the lower curve the flow velocity profile. This figure is for a supersonic spherical deflagration bubble with an initial energy density $\epsilon_{f}=100 B$ and shock velocity $\xi_{\text {sh }}=0.625$. The velocity of the phase transition front is $\xi_{\text {defl }}=0.6163$.

FIG. 5. The energy densities (a) and flow velocities (b) at special points of the bubble profile (see Fig. 4). These quantities are shown as a function of the phase transition velocity $\xi_{\text {p.t. }}$ for a fixed initial energy density $\epsilon_{f}=100 B$. The solution switches from a weak deflagration bubble to a supersonic deflagration bubble at $\xi_{\text {p.t. }}=c_{s}=0.57735$ and to a weak detonation bubble at $\xi_{\text {p.t. }}=\xi_{\text {det }, \mathrm{J}}=0.6534$.

FIG. 6. The special points of the bubble profile as a function of $\xi_{\text {p.t. }}$ for $\epsilon_{f}=100 B$. A weak deflagration bubble has a region of shocked fluid between $\xi=\xi_{\text {defl }}$ and $\xi=\xi_{\text {sh }}$. A weak detonation bubble has a rarefaction wave extending from $\xi=c_{s}$ to $\xi=\xi_{\text {det }}$. A supersonic deflagration bubble has both features.

FIG. 7. The velocity $\xi_{\text {det,J }}$ of the phase transition front in the Jouguet detonation bubble and the shock velocity $\xi_{\mathrm{sh}, \mathrm{J}}$ in the Jouguet deflagration bubble as a function of the initial energy density $\epsilon_{f}$. As the velocity of a weak deflagration is increased from 0 to $c_{s}$, the shock velocity increases from $c_{s}$ to $\xi_{\mathrm{sh}, \mathrm{J}}$, at which point the solution becomes a supersonic deflagration bubble. As the deflagration velocity is further increased from $c_{s}$ to $\xi_{\text {det,J }}$ the shock velocity increases from $\xi_{\text {sh,J }}$ to $\xi_{\text {det,J }}$ at which point the phase transition front catches up with the shock and the solution becomes a detonation bubble. 


\author{
H. Kurki-Suonio ${ }^{a *}$ and M. Laine ${ }^{b \dagger}$ \\ ${ }^{a}$ Research Institute for Theoretical Physics and ${ }^{b}$ Department of Physics, \\ P.O. Box 9, FIN-00014 University of Helsinki, Finland
}

(January 5, 1995)

\begin{abstract}
The classification of the hydrodynamical growth mechanisms for the spherical bubbles of the low-temperature phase in cosmological phase transitions is completed by showing that the bubbles can grow as supersonic deflagrations. Such deflagrations consist of a Jouguet deflagration, followed by a rarefaction wave. Depending on the amount of supercooling, the maximal velocity of supersonic deflagrations varies between the sound and the light velocities. The solutions faster than supersonic deflagrations are weak detonations.
\end{abstract}

PACS numbers: 98.80.Cq, 47.75.+f, 95.30.Lz

\section{INTRODUCTION}

When an exothermic process takes place in an ideal fluid, knowing the hydrodynamics of growing bubbles becomes relevant. Such situations include spherically symmetric chemical burning in combustible gases $[1,2]$, and the growth of bubbles of the low-temperature phase in cosmological first-order phase transitions [3-13]. The solutions of the energy-momentum conservation equations across the 'burning' surface can naturally be divided into six hydrodynamical modes with different qualitative features, called the weak, strong, and Jouguet deflagrations, and the weak, strong, and Jouguet detonations. When stability considerations, the microscopic mechanism of entropy production at the burning surface, and the overall boundary conditions, are taken into account, it turns out that some of the hydrodynamical modes are actually forbidden. It is the purpose of the present paper to complete the existing classification of the allowed hydrodynamical modes in cosmological phase transitions, and to present, to our knowledge, a new flow profile by which the burning surface can be connected to the boundary conditions.

Our new results concern deflagrations, which are the slower of the two main types of solutions. Usually, it is assumed that the flow profile of a deflagration bubble is such that the burning surface leaves the matter at rest. Then weak deflagrations are subsonic and Jouguet deflagrations expand at sound velocity. The strong deflagrations would be supersonic, but it is known for chemical

*Email: hkurkisu@pcu.helsinki.fi

'Email: mlaine@phcu.helsinki.fi burning that such solutions do not exist $[1,2]$. We find that the strong deflagrations are forbidden in cosmological phase transitions, as well. However, there is another type of a supersonic mode. Indeed, a Jouguet deflagration can also leave behind it a rarefaction wave, in which case matter is not at rest immediately behind the burning surface. Such solutions are supersonic, and fill in the velocity gap between the sound velocity and the slowest detonations. As far as we can see, supersonic deflagrations should be possible both in chemical burning, and in cosmological phase transitions, such as the QCD and the electroweak phase transition.

The plan of the paper is the following. In Sec. II, the properties of bubble growth in relativistic ideal fluid are reviewed both for deflagrations and detonations. In Sec. III, we use a simple dynamical model to study which hydrodynamical growth mechanisms can actually be reached from an initial critical bubble configuration. In particular, supersonic deflagrations are found to be possible. The structure of spherical supersonic deflagration bubbles is analyzed in detail in Sec. IV, for the bag equation of state. The conclusions are in Sec. V.

\section{DEFLAGRATIONS AND DETONATIONS}

Let us go into the rest frame of the expanding phase transition surface. Imposing energy-momentum conservation across the discontinuity gives the equations

$$
\begin{aligned}
w_{q} \gamma_{q}^{2} v_{q} & =w_{h} \gamma_{h}^{2} v_{h} \\
w_{q} \gamma_{q}^{2} v_{q}^{2}+p_{q} & =w_{h} \gamma_{h}^{2} v_{h}^{2}+p_{h}
\end{aligned}
$$

where the subscripts $q$ and $h$ refer to the inflowing high-temperature ('quark') phase and the out-flowing low-temperature ('hadron') phase, respectively ( $w$ is the proper enthalpy density). We choose the signs so that the velocities are positive, and denote by $F$ the quantity $w_{q} \gamma_{q}^{2} v_{q}$. It then follows that

$$
F=\frac{p_{h}-p_{q}}{v_{q}-v_{h}}>0 .
$$

This equation tells us that there are two qualitatively different kinds of solutions. For deflagrations, the pressure decreases and the velocity increases across the surface: $p_{h}<p_{q}$ and $v_{h}>v_{q}$. For detonations, the opposite is true. For deflagrations, the inflow velocity $v_{q}$ is subsonic, and for detonations, it is supersonic $[1,14]$. Note that for 
deflagrations, the sign of the pressure difference is opposite to what it would have been, had the temperature not changed across the phase transition surface.

A further division of the different hydrodynamical processes can be made according to the outflow velocity $v_{h}$. Both for deflagrations and detonations, energymomentum conservation allows $v_{h}$ to be on either side of, or equal to, the sound velocity $c_{s}$. If $v_{h}$ equals $c_{s}$, the process is called a Jouguet process. If $v_{h}$ is on the same side of $c_{s}$ as $v_{q}$, the process is called weak; otherwise it is called strong. For instance, for weak deflagrations, $v_{h}<c_{s}$.

To construct a complete bubble solution, the discontinuity at the phase transition surface has to be matched with possible shock discontinuities and continuous flow profiles, to satisfy the boundary conditions. A deflagration solution can be constructed from a supersonic shock front preceding the phase transition surface and setting the cosmic matter moving, followed by a continuous flow profile, and terminated by the phase transition front bringing the matter back at rest. A detonation solution can be constructed from a weak or Jouguet detonation front moving supersonically into matter at rest, and followed by a continuous rarefaction wave bringing the matter again at rest. With a strong detonation, the boundary conditions of a spherically expanding bubble cannot be satisfied $[1,3]$. Flow profiles of deflagration bubbles are given in Ref. [5], and flow profiles of detonation bubbles are illustrated, e.g., in Fig. 2 of Ref. [14]. These simplest complete flow profiles are dubbed, according to the nature of the phase transition front, the weak, Jouguet and strong deflagrations, and the weak and Jouguet detonations. In the next Section, it is seen that the presented solutions do not quite exhaust the flow profiles relevant for cosmological phase transitions. In the remainder of this Section, on the other hand, it is argued that some of the solutions will not be realized in nature.

First, strong deflagrations are expected to be forbidden. For chemical combustion, there are at least two ways of seeing this. Assuming that the burning front can be described as a changing mixture of the burnt and the unburnt matter, one can see that strong deflagrations would include regions of negative entropy production $[2,14]$, and they are therefore impossible. This proof is not valid for cosmological phase transitions, since the assumption of a changing mixture would lead to a vanishing surface tension at $T_{c}$. There is another way of excluding the strong deflagrations, coming from the observation that even if such a solution could momentarily be forced to exist, it would be unstable and tend to split into other solutions [1]. This argument should hold also for cosmological phase transitions. Hence we expect to see only weak and Jouguet deflagrations. These expand at most at sound velocity.

Second, consider weak detonations. To study them, one must know something about the microscopic mechanism of phase separation. For chemical combustion $[1,2]$, the rate of burning rises dramatically with temperature, and the velocity of the burning front is determined by how fast heat can be supplied to yet unburnt regions. As a consequence, the microscopic structure of a detonation front is such that a shock front heats the matter so much that rapid burning can begin, and a layer where the burning takes place follows just behind the shock front ${ }^{1}$. For weak detonations, the layer behind the shock front is a strong deflagration. Hence, the impossibility of strong deflagrations implies the impossibility of weak detonations. For cosmological phase transitions, the structure of the burning front is different. A phase transition is more apt to start at a low temperature, when supercooling is larger. In particular, a point in space outside the original critical bubble does not wait for a rise in temperature to start transforming into the new phase, but the transformation is caused by interactions with the new phase in the detonation front. Hence, the structure of a detonation front need not be a shock front followed by a deflagration, and weak detonations cannot be excluded in cosmological phase transitions [14].

Let us note that there exist processes in classical hydrodynamics which have the same classification of the different hydrodynamical growth mechanisms as cosmological phase transitions, unlike chemical burning. Such are condensation discontinuities $[1, \$ 132]$. An example of a condensation discontinuity is the condensation of supersatured water vapour into small water droplets, i.e., fog. This is a phase transition, though with a finite chemical potential. Indeed, only strong deflagrations and detonations are excluded in Ref. [1], and the other processes are accepted.

\section{NUMERICAL RESULTS}

To be able to check the presented arguments about the possibility of the different kinds of solutions, one would like to have a way of taking automatically into account energy-momentum conservation, boundary conditions, and the microscopic structure of the phase transition surface. One might also wish to follow the real-time history of the growing bubbles, to see which stationary final states can actually be reached from reasonable initial conditions. These problems can be solved by imposing an equation of motion for some continuously changing

\footnotetext{
${ }^{1}$ This assumption was also made by Steinhardt in Ref. [3]. In Ref. [3] weak detonations were excluded from consideration by the statement "The lower point $b$ at which the chord ad intersects the detonation adiabat cannot be reached if the reaction is exothermic", which assumes the detonation is initiated by a shock. Ref. [3] is often quoted as a proof that Jouguet detonations are the only kinds of detonations that occur, but this is a result of relativistic combustion theory, which does not apply to cosmological phase transitions.
} 
order parameter field $\varphi(t, \mathbf{x})$, and by then solving the equation. The equation could, in principle, be derived from non-equilibrium field theory.

In Ref. [15], the simple phenomenological form

$$
\partial^{\mu} \partial_{\mu} \varphi+\frac{\partial V}{\partial \varphi}+\eta u^{\mu} \partial_{\mu} \varphi=0
$$

of the equation for the $\varphi$-field was proposed (in Ref. [15] the notation $1 / \Gamma$ was used for $\eta)$. This is the simplest relativistic generalization of the Langevin equation without the noise term. The parameter $\eta$ fixes the one degree of freedom left free by energy-momentum conservation equations. From eq. (4) and energy-momentum conservation it follows that the entropy density $s$ satisfies

$$
T \partial_{\mu}\left(s u^{\mu}\right)=\eta\left(u^{\mu} \partial_{\mu} \varphi\right)^{2},
$$

so that $\eta$ can be interpreted as a parametrization of the entropy production at the phase transition surface. Below, $\eta$ is treated as a free parameter, although for the EW phase transition, its value could be estimated [15] by comparing the analytic formula for the deflagration front velocity in the limit of small supercooling with microscopic calculations [16-18] of the same quantity.

In Ref. [15], eq. (4) and the energy-momentum conservation equations were solved numerically for the planar symmetric case, corresponding to a $1+1$ dimensional spacetime. Note that the dimension of space $d$ does not affect the results qualitatively, since the phase transition front looks locally planar, and the qualitative features of the continuous flow profiles outside the phase transition front do not depend on $d$ [5]. There are two methods of solving eq. (4). Either one can take some initial bubble, and integrate all the partial differential equations forward in time (the dynamical code), or one can search only for stationary final states (the static code).

The general structure of the stationary final states is shown in Fig. 1. In this figure, three kinds of solutions are displayed. First, the dotted lines $(\alpha, \ldots)$ each represent a one-parameter family of solutions, with a given initial supercooling $T_{f}$, which satisfy the energy-momentum conservation equations and the boundary conditions, alone. In drawing these curves, it was assumed that the solutions are of the type discussed in Sec. II, Second, the solid lines represent the solutions which satisfy energymomentum conservation and eq. (4) across the phase transition surface, with a given $\eta$, but for which the global boundary conditions have not been imposed. For given $T_{f}$ and $\eta$, the possible stationary final states are then found from the intersections of these two kinds of curves. Third, the gray lines indicate those stationary final states which are actually realized by the dynamical code, starting from a reasonable initial state, for given $T_{f}$, as a function of $\eta$. As illustrated with the arrows for the curve $\alpha$, the solution is a weak deflagration in the limit $\eta \rightarrow \infty$, moves towards strong deflagrations as $\eta$ is decreased, and changes to a detonation at some point.

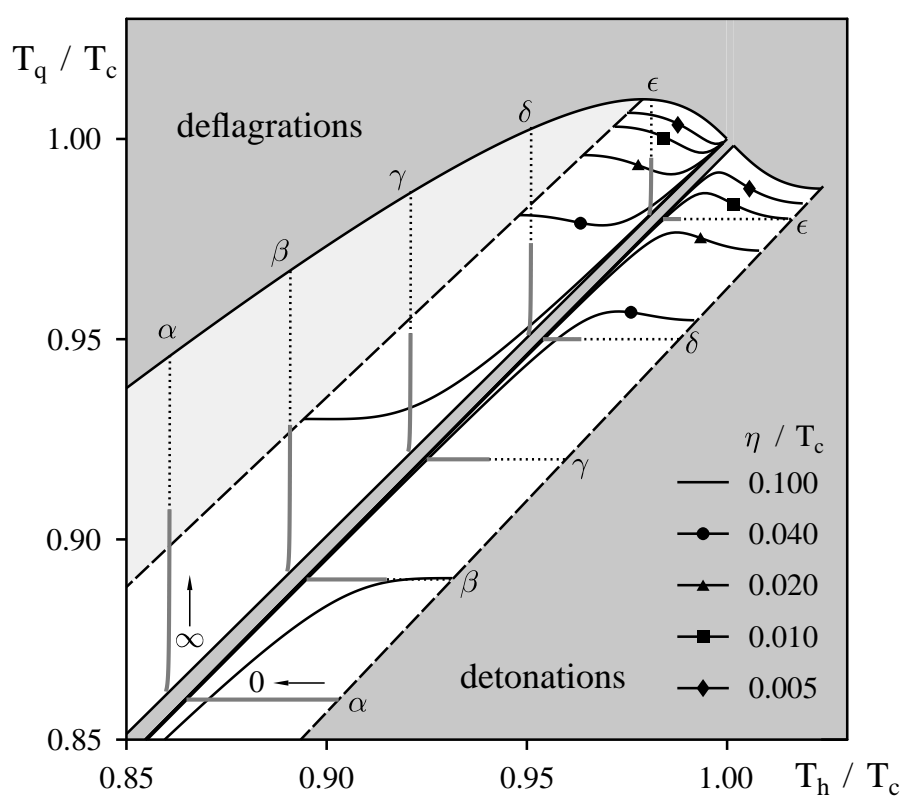

FIG. 1. The solutions of the model of Ref. [15], in the case of planar symmetry. In $1+3$ dimensions, the solid lines remain the same, but the exact functional form of the dotted lines changes a little. For detonations, the $x$-axis is the temperature just behind the phase transition surface; for deflagrations, it is the temperature in the center of the expanding bubble. The $y$-axis is the temperature just in front of the phase transition surface. The region forbidden kinematically, by the non-negativity of entropy production, or by boundary conditions, has been covered with the darker shade, assuming the types of solutions discussed in Sec. II. The two allowed regions represent the deflagrations and the weak detonations. Dashed lines indicate the Jouguet processes, and strong deflagrations are covered with the lighter shade. The meaning of the different curves has been explained in the text. The nucleation temperatures $T_{f} / T_{c}$ corresponding to $\alpha, \beta, \gamma, \delta$ and $\epsilon$ are $0.86,0.89,0.92,0.95$ and 0.98 . The (QCD-type) parameters used in this figure are $L=0.1 T_{c}^{4}, \sigma=0.1 T_{c}^{3}$ and $l_{c}=6 T_{c}^{-1}$. Given the expansion rate of the Universe, the actual nucleation temperature $T_{f} / T_{c}$ is then about 0.86 (Fig. 1 in Ref. [19]).

A few interesting things can be seen in Fig. 1. First, one should note the general feature that weak detonations exist, as anticipated in Sec. II. Second, the dotted lines have a very large gradient for deflagrations. This indicates that the temperature $T_{q}$ in front of the phase transition surface may be much higher than $T_{f}$ due to the heating caused by the shock front, but the temperature $T_{h}$ behind the phase transition surface is very near $T_{f}$. For detonations, similarly, it turns out that the temperature in the center of the expanding bubble differs much less from $T_{f}$ than the temperature $T_{h}$ just behind the detonation front. Third, it is seen that for some $T_{f}$ and $\eta$, there are more than one possible stationary final 
states. For instance, for $\eta=0.010 T_{c}$ and $T_{f}=0.98 T_{c}$, there are three possible final states. The dynamical code selects one of these ${ }^{2}$. The solutions which are not realized have a sharp peak in temperature expanding at roughly the speed of sound. If one gives the unreached weak deflagration as an initial condition to the dynamical code, it remains as such, indicating that this solution may be reachable from other initial conditions. However, the unreached weak detonation transforms to either of the other solutions, suggesting an instability.

Let us now turn to the existence of supersonic deflagrations. From Fig. 1, it is seen that strong deflagrations have not been found with the static code. Technically, this is due to the fact that no matter how well one tries to guess the correct solution, the guess does not relax to a strong deflagration but rather changes considerably to form a weak detonation, with the same $T_{h}$. This is in accordance with strong deflagrations being unstable, as argued in the previous Section. On the other hand, with the dynamical code, we have found supersonic solutions which lie very near the dotted lines representing strong deflagrations (see curve $\alpha$ ). A careful inspection reveals, however, that in the region covered by the lighter shade, the gray curve is not exactly on the dotted curve. By drawing a figure of the temperature profiles, the reason becomes apparent. In Fig. 2, temperature profiles are shown for the supercooling $T_{f}=0.80 T_{c}$. The supersonic deflagration solution $\left(\eta=0.17 T_{c}\right)$ is seen to be comprised of a shock front, a phase transition front, and a rarefaction wave in the low-temperature phase. If the temperature just behind the phase transition front is taken as the $x$-coordinate in Fig. 1 instead of the temperature in the center of the bubble, the solution lies on the dashed curve corresponding to a Jouguet deflagration. Furthermore, if one gives a strong deflagration as an initial condition to the dynamical code, the solution is unstable, and transforms into a Jouguet deflagration followed by a rarefaction wave. In the next Section, these solutions are analyzed in more detail in $1+3$ dimensions.

\footnotetext{
${ }^{2}$ Figs. 19 and 20 of Ref. [15] represent one solution of the static code. The solution of the dynamical code actually changes into a weak detonation at around $\eta=0.03 T_{c}$, with $v_{\text {det }} \approx 0.7$.
}

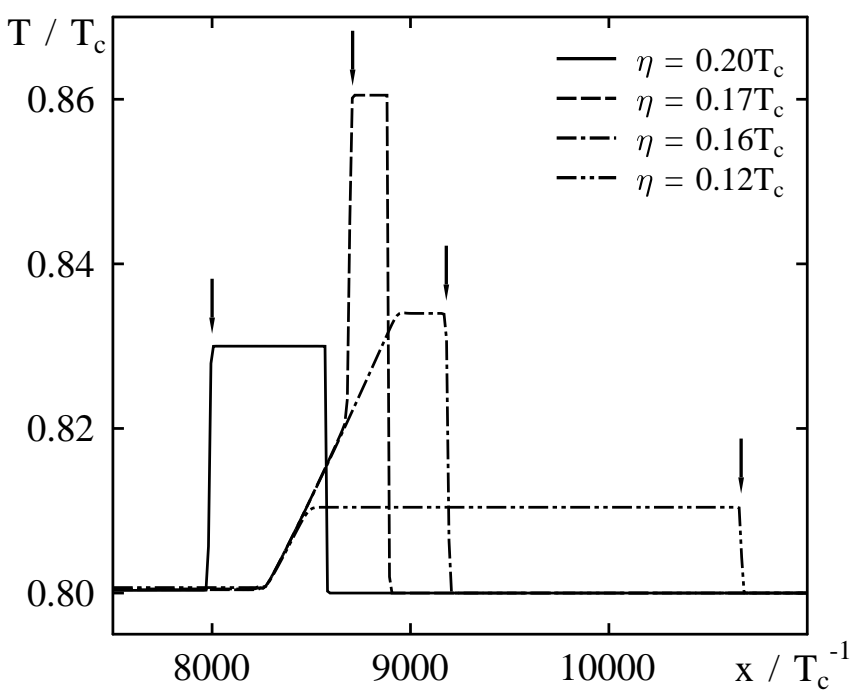

FIG. 2. Temperature profiles at $t=14400 T_{c}^{-1}$ for different values of $\eta$. All the profiles are moving to the right. The arrows indicate the location of the phase transition front. For $\eta=0.20 T_{c}$, the solution is a weak deflagration; for $\eta=0.17 T_{c}$, it is a Jouguet deflagration followed by a rarefaction wave; for $\eta=0.16 T_{c}$ and $\eta=0.12 T_{c}$, the solution is a weak detonation. The velocity of the phase transition front changes from 0.56 at $\eta=0.20 T_{c}$ to 0.74 at $\eta=0.12 T_{c}$.

\section{SPHERICAL SOLUTIONS}

For an overview of the different hydrodynamical solutions for spherical phase transition bubbles, we employ a simpler equation of state, the bag EOS

$$
\begin{aligned}
& p_{h}=\frac{1}{3} \epsilon_{h}, \\
& p_{q}=\frac{1}{3}\left(\epsilon_{q}-4 B\right),
\end{aligned}
$$

where $B$ is the bag constant ( $\epsilon$ is the proper energy density). We assume a spherically symmetric similarity solution, i.e., it can be described in terms of the single coordinate $\xi \equiv r / t$. In contrast to the previous Section, we are here not interested in how the bubble relaxes to the similarity solution from some initial condition. We also give up modelling the microscopic structure of the phase transition front, and treat it as a discontinuity. Therefore we do not have a parameter $\eta$ coming from microphysics to select among the different solutions hydrodynamically allowed. We just parametrize the solutions by the velocity of the phase transition front $\xi_{\text {p.t. }}$ ( $\xi_{\text {defl }}$ or $\left.\xi_{\text {det }}\right)$. Then we get almost analytical (i.e., involving ordinary differential equations) solutions for the bubble structure, instead of having to resort to numerical hydrodynamics.

The continuous parts of the flow satisfy the equations $[5]$ 


$$
\left[v^{2}\left(3-\xi^{2}\right)-4 v \xi+3 \xi^{2}-1\right] \frac{d v}{d \xi}=2 \frac{v}{\xi}\left(1-v^{2}\right)(1-\xi v)
$$

and

$$
\frac{1}{\tilde{\epsilon}} \frac{d \tilde{\epsilon}}{d \xi}=4 \frac{\xi-v}{1-\xi v} \frac{1}{1-v^{2}} \frac{d v}{d \xi}
$$

where $\tilde{\epsilon}=\epsilon$ in the $h$ phase and $\tilde{\epsilon}=\epsilon-B$ in the $q$ phase.

In Fig. 3 we show a sequence of profiles for phase transition bubbles, with the same initial energy density $\epsilon_{f}$, but for different $\xi_{\text {p.t. }}$.

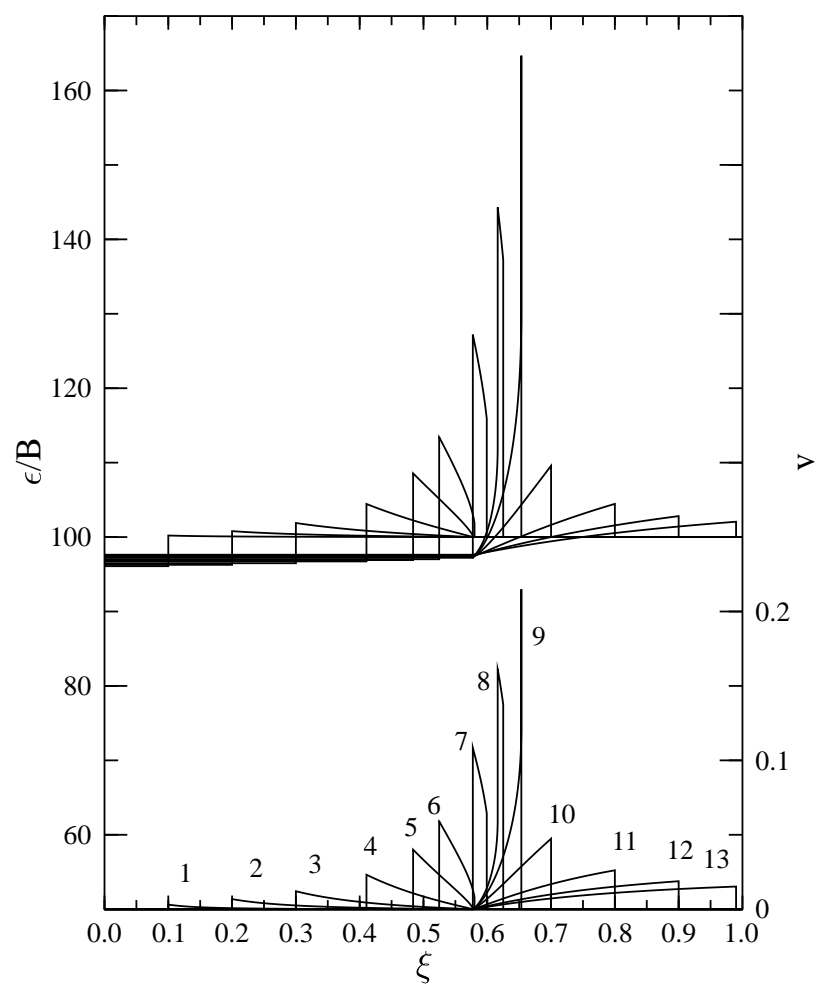

FIG. 3. Energy-density (upper set) and flow velocity (lower set) profiles for spherical phase transition bubbles. We show 13 profiles, all for the same initial energy density $\epsilon_{f}=100 B$, but with different phase transition front velocities. The six slowest (on the left) are weak deflagrations. The seventh is a Jouguet deflagration bubble, with $\xi_{\text {defl }}=c_{s}$. The eighth is a supersonic deflagration bubble, which has a Jouguet deflagration front preceded by a shock and followed by a rarefaction wave. The ninth is a supersonic deflagration bubble almost at the limit where it becomes a Jouguet detonation. The shocked region is now so thin that it appears as a mere vertical line in the figure. The last four (on the right) are weak detonations.

If the phase transition front is subsonic, $\xi_{\text {p.t. }}=\xi_{\text {defl }} \leq$ $c_{s}$, we have a deflagration bubble of the type described in [5]. The phase transition front is preceded by a shock at $\xi_{\text {sh }}>c_{s}$, the matter is at rest at $\xi>\xi_{\text {sh }}$ and at $\xi<\xi_{\text {defl }}$, and the shocked fluid in between has a continuous flow profile, with the (outward) flow velocity and energy density increasing with decreasing $\xi$. We denote by $\xi_{\text {sh,J }}$ the shock velocity of the Jouguet deflagration bubble with $\xi_{\text {p.t. }}=c_{s}$. The slowest deflagrations have extremely weak shocks at $\xi_{\text {sh }} \sim c_{s}$.

The slowest detonation is the Jouguet detonation, with

$$
\xi_{\mathrm{det}, \mathbf{J}}=\frac{1+3 \sqrt{\frac{B}{2 \epsilon_{f}+B}}}{1+\sqrt{\frac{B}{2 \epsilon_{f}+B}}} c_{s} .
$$

Solutions with $\xi_{\text {p.t. }}>\boldsymbol{\xi}_{\text {det,J }}$ are weak detonations.

The velocity range between $\xi_{\text {p.t. }}=c_{s}$ and $\xi_{\text {p.t. }}=\xi_{\text {det, J }}$ is covered by the supersonic deflagrations. As $\xi_{\text {p.t. }}$ is increased past $c_{s}$, a rarefaction wave appears in the region between $\xi=c_{s}$ and $\xi=\xi_{\text {p.t. }}$. The phase transition remains a Jouguet deflagration, and is preceded by a shock, with $\xi_{\text {p.t. }}<\xi_{\text {sh }}<\xi_{\text {det,J. }}$. As $\xi_{\text {p.t. }}$ is increased, $\xi_{\text {sh }}$ grows also, but not as rapidly, and thus the region of shocked fluid becomes narrower. The two velocities $\xi_{\text {p.t. }}$ and $\xi_{\text {sh }}$ become equal at $\xi_{\text {p.t. }}=\xi_{\text {sh }}=\xi_{\text {det }, \mathrm{J}}$, the region of shocked fluid disappears and the solution becomes a Jouguet detonation.

In the limit where $\xi_{\text {p.t. }}$ approaches $\xi_{\text {det,J }}$ from below, the shocked region becomes infinitesimally thin. This resembles the microscopic structure of a detonation front in chemical combustion, where heating by a shock initiates the transition. However, the weak detonations $\left(\xi_{\text {p.t. }}>\xi_{\text {det }, J}\right)$ here do not have such structure.

In Fig. 4 we show the structure of a supersonic deflagration bubble. Figs. 5 and 6 show how the different quantities in the bubble change as a function of $\xi_{\text {p.t. }}$. Fig. 7 shows the limiting velocities $\xi_{\text {sh }, \mathbf{J}}$ and $\xi_{\text {det, }}$ as a function of $\epsilon_{f} / B$.

Let us note that in this Section we have studied pure hydrodynamics, whereas one should actually also insist on entropy production being non-negative at the phase transition front [4]. This requires knowledge of the dimensionless parameter $r=a_{q} / a_{h}$ in the bag EOS $\left(\epsilon_{h}=3 a_{h} T^{4}, \epsilon_{q}=3 a_{q} T^{4}+B\right)$. The parameter $r$ fixes the energy density $\epsilon / B=(4 r-1) /(r-1)$ corresponding to the critical temperature $T_{c}$. The entropy condition restricts the availability of the different hydrodynamical modes near $T_{c}$, and for a sufficiently small supercooling, only weak deflagrations remain allowed [19, Sec. IV]. However, considering any $\epsilon_{f}$, for a sufficiently small $r$ this value of $\epsilon_{f}$ will correspond to a large supercooling, and all the hydrodynamical modes are allowed. Therefore the entropy condition does not change our conclusions. 


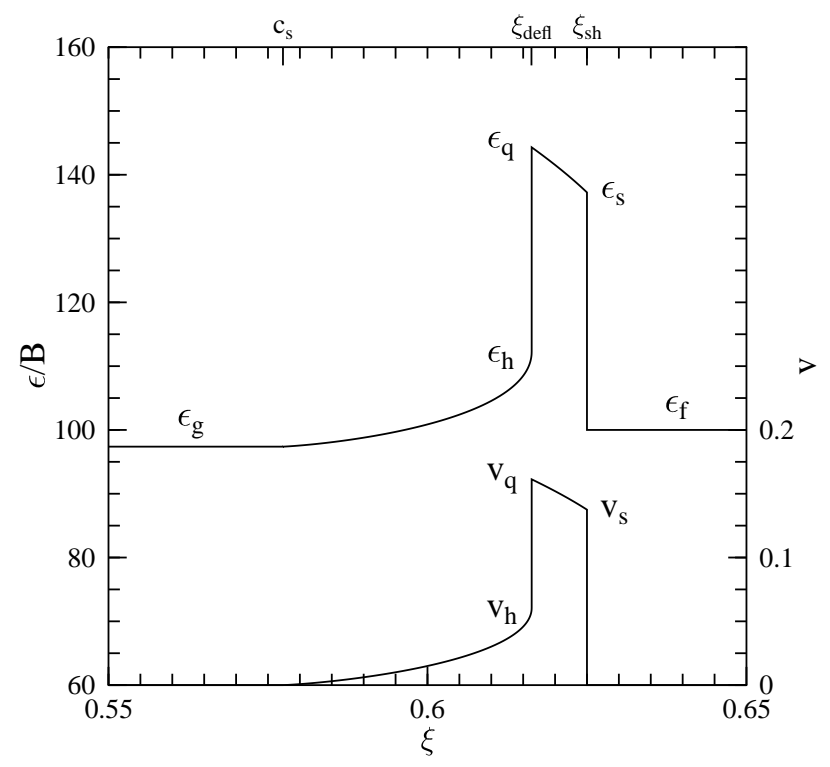

FIG. 4. A supersonic deflagration bubble. The upper curve is the energy density profile and the lower curve the flow velocity profile. This figure is for a supersonic spherical deflagration bubble with an initial energy density $\epsilon_{f}=100 B$ and shock velocity $\xi_{s h}=0.625$. The velocity of the phase transition front is $\xi_{\mathrm{defl}}=0.6163$.
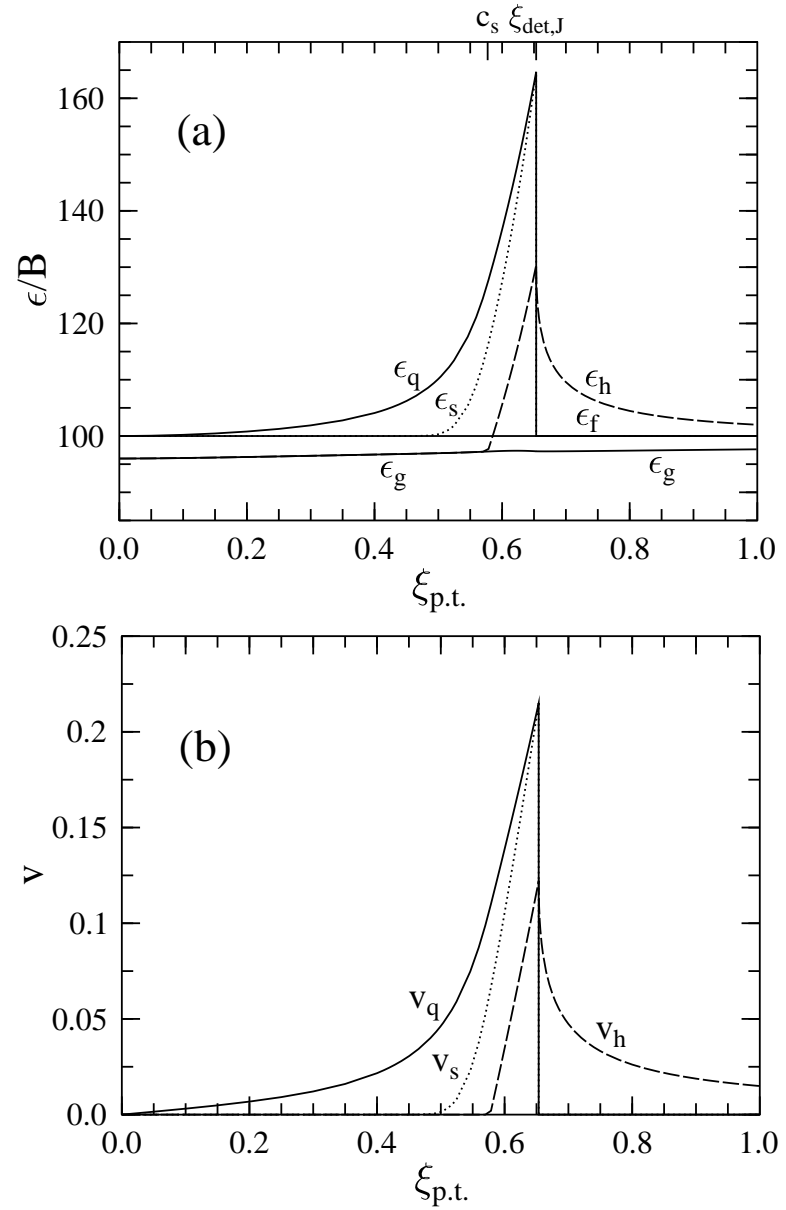

FIG. 5. The energy densities (a) and flow velocities (b) at special points of the bubble profile (see Fig. 4). These quantities are shown as a function of the phase transition velocity $\xi_{\text {p.t. }}$ for a fixed initial energy density $\epsilon_{f}=100 B$. The solution switches from a weak deflagration bubble to a supersonic deflagration bubble at $\xi_{\mathrm{p} . t .}=c_{s}=0.57735$ and to a weak detonation bubble at $\xi_{\text {p.t. }}=\xi_{\text {det }, \mathrm{J}}=0.6534$. 


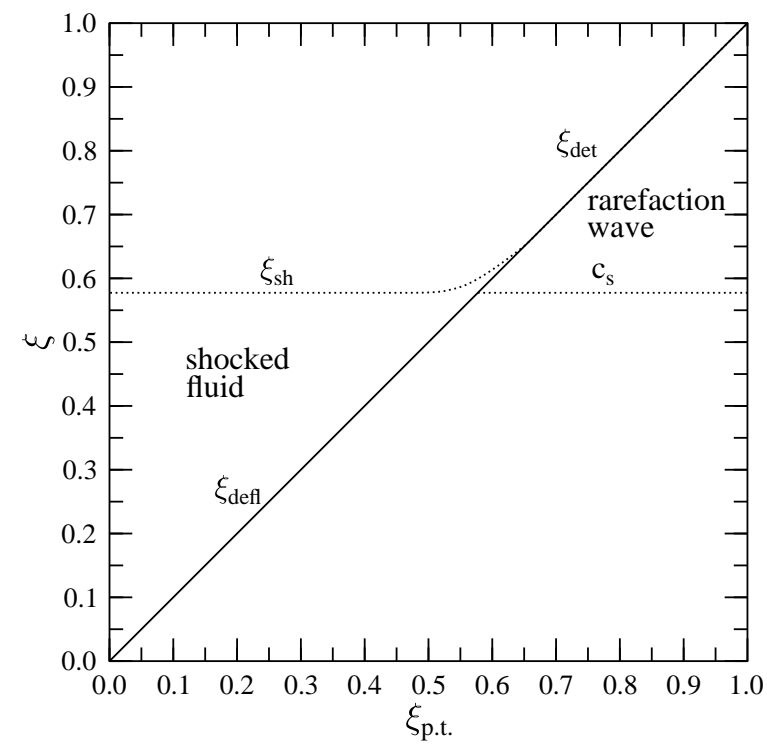

FIG. 6. The special points of the bubble profile as a function of $\xi_{\mathrm{p} . t .}$ for $\epsilon_{f}=100 \mathrm{~B}$. A weak deflagration bubble has a region of shocked fluid between $\xi=\xi_{\text {defl }}$ and $\xi=\xi_{\mathrm{sh}}$. A weak detonation bubble has a rarefaction wave extending from $\xi=c_{s}$ to $\xi=\xi_{\mathrm{det}}$. A supersonic deflagration bubble has both features.

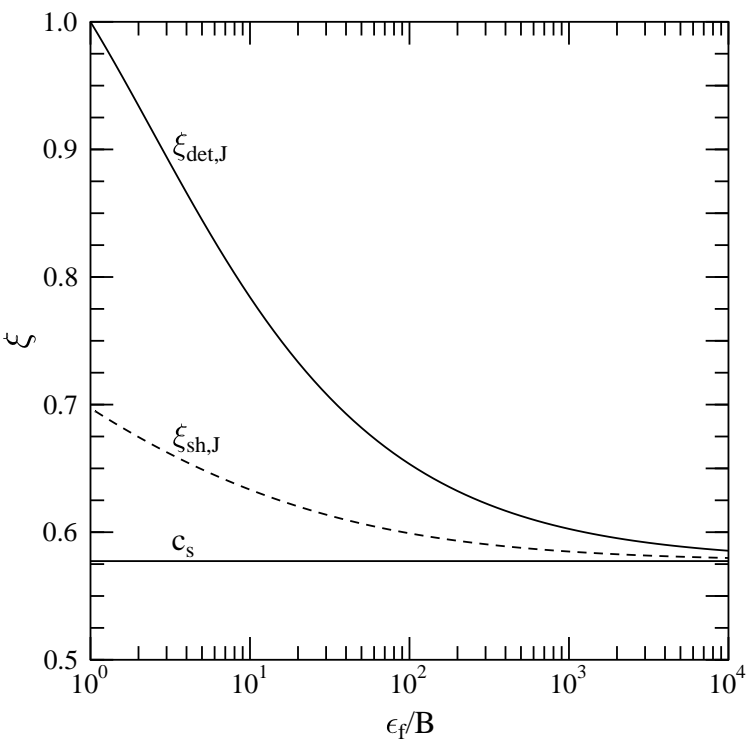

FIG. 7. The velocity $\xi_{\text {det }, \mathrm{J}}$ of the phase transition front in the Jouguet detonation bubble and the shock velocity $\xi_{\mathrm{sh}, \mathrm{J}}$ in the Jouguet deflagration bubble as a function of the initial energy density $\epsilon_{f}$. As the velocity of a weak deflagration is increased from 0 to $c_{s}$, the shock velocity increases from $c_{s}$ to $\xi_{\mathrm{sh}, \mathrm{J}}$, at which point the solution becomes a supersonic deflagration bubble. As the deflagration velocity is further increased from $c_{s}$ to $\xi_{\text {det }, \mathrm{J}}$ the shock velocity increases from $\xi_{\mathrm{sh}, \mathrm{J}}$ to $\xi_{\text {det,J }}$ at which point the phase transition front catches up with the shock and the solution becomes a detonation bubble.

\section{CONCLUSIONS}

We have discovered a new kind of a bubble solution for cosmological phase transitions. The phase transition front of such a bubble is a Jouguet deflagration, is preceded by a shock, and followed by a rarefaction wave. The phase transition front moves supersonically with respect to the matter at rest. These supersonic deflagration bubbles fill the velocity gap between weak deflagrations, which are subsonic, and weak detonations. The two limiting cases of the supersonic deflagration bubble are the 'ordinary' Jouguet deflagration bubble (i.e., without a rarefaction wave) and the Jouguet detonation bubble.

It has been known that strong deflagrations would not occur since they are unstable to 1-dimensional perturbations (i.e., in the radial direction). By following a strong deflagration bubble with a numerical hydrodynamics code we have seen how such a perturbation transforms the strong deflagration into a Jouguet deflagration by developing a rarefaction wave. This gives rise to the supersonic deflagration bubble we have described.

By modelling the microscopic mechanism of entropy production at the phase transition front by a simple dynamical model, we found that the solutions 'near' Jouguet detonations [e.g., profiles $7-9$ in Fig. 3] were in many cases not realized in a numerical evolution, although they were allowed by the external conditions (including the entropy condition). The velocity $\xi_{\text {p.t. }}$ of the phase transition front was found not to increase continuously as the microscopic entropy-production parameter $\eta$ was decreased, but the solution jumped from a weak deflagration to a much faster weak detonation. Thus the most 'violent' solutions, i.e., the ones with large energy densities and flow velocities occurring near the phase transition front, were skipped over. This happened in the cases with a small supercooling, and we had to go to large supercooling to see examples of supersonic deflagrations. Examining Fig. 1, one finds for small supercooling that, for a given value of $T_{f}$ and $\eta$ there can be as many as three solutions allowed, not just one. One of these is a deflagration, and two are detonations. In the numerical evolution, the weaker, i.e., the faster, of the detonations was then realized.

This suggests that in nature the more dramatic solutions, the Jouguet detonation and solutions near it, are 
more difficult to realize, especially for small supercooling. This is in sharp contrast to chemical burning, where the Jouguet detonations are the only possible detonation solutions.

\section{ACKNOWLEDGEMENTS}

We are grateful to J. Ignatius for discussions, and to the Finnish Center for Scientific Computing for computational resources.

[1] L. D. Landau and E. M. Lifshitz, Fluid Mechanics, 2nd edition (Pergamon Press, Oxford, 1987).

[2] R. Courant and K. O. Friedrichs, Supersonic flow and shock waves (Springer-Verlag, Berlin, 1985).

[3] P. J. Steinhardt, Phys. Rev. D 25 (1982) 2074.

[4] M. Gyulassy, K. Kajantie, H. Kurki-Suonio, and L. McLerran, Nucl. Phys. B 237 (1984) 477.

[5] H. Kurki-Suonio, Nucl. Phys. B 255 (1985) 231.

[6] K. Kajantie and H. Kurki-Suonio, Phys. Rev. D 34 (1986) 1719.

[7] J. C. Miller and O. Pantano, Phys. Rev. D 40 (1989) 1789, D 42 (1990) 3334.

[8] K. Enqvist, J. Ignatius, K. Kajantie, and K. Rummukainen, Phys. Rev. D 45 (1992) 3415.

[9] S. A. Bonometto and O. Pantano, Phys. Rep. 228 (1993) 175.

[10] P. Huet, K. Kajantie, R.G. Leigh, B.-H. Liu, and L. McLerran, Phys. Rev. D 48 (1993) 2477.

[11] M. Kamionkowski, A. Kosowsky, and M. S. Turner, Phys. Rev. D 49 (1994) 2837.

[12] A. F. Heckler, preprint astro-ph/9407064 (1994).

[13] J. C. Miller and L. Rezzolla, SISSA preprint 161/94/AEP (1994).

[14] M. Laine, Phys. Rev. D 49 (1994) 3847.

[15] J. Ignatius, K. Kajantie, H. Kurki-Suonio, and M. Laine, Phys. Rev. D 49 (1994) 3854.

[16] M. Dine, P. Huet, R.G. Leigh, A.D. Linde, and D. Linde, Phys. Rev. D 46 (1992) 550.

[17] B.-H. Liu, L. McLerran, and N. Turok, Phys. Rev. D 40 (1992) 2668.

[18] S.Yu. Khlebnikov, Phys. Rev. D 46 (1992) 3223.

[19] J. Ignatius, K. Kajantie, H. Kurki-Suonio, and M. Laine, Phys. Rev. D 50 (1994) 3738. 